\title{
Prospects for Advanced RF Theory and Modeling
}

\author{
D. B. Batchelor \\ Oak Ridge National Laboratory, Oak Ridge, Tennessee 37831-8071
}

\section{INTRODUCTION}

This paper represents an attempt to express in print the contents of a rather philosophical review talk. The charge for the talk was not to summarize the present status of the field and what we can do, but to assess what we will need to do in the future and where the gaps are in fulfilling these needs. The objective was to be complete, covering all aspects of theory and modeling in all frequency regimes, although in the end the talk mainly focussed on the ion cyclotron range of frequencies (ICRF). In choosing which areas to develop, it is important to keep in mind who the customers for RF modeling are likely to be and what sorts of tasks they will need for RF to do. This occupies the first part of the paper. Then we examine each of the elements of a complete RF theory and try to identify the kinds of advances needed.

\section{FUTURE CUSTOMERS}

An important challenge for the fusion program will be to learn to operate in "advanced" confinement regimes as defined, for example, by the concept of the Advanced Tokamak. An advanced tokamak is typically thought of as one which achieves economical attractiveness through improved performance relative to a conventional tokamak: 1) smaller size, higher fusion power density through increased $\beta$ and $\tau_{E} ; 2$ ) reduced recirculating power through increased bootstrap current fraction and improved current drive efficiency; 3) smaller net current for reduced susceptibility to disruptions and; 4) steady state. These are not simply idle hopes. A number of approaches to improved confinement and stability have been demonstrated, at least for limited periods of time - H-modes, VH-modes, reverse shear, high internal inductance, and edge radiative improved modes. Improved performance must be achieved by carefully controlling a number of complicated, non-linearly interacting plasma processes: collisional and turbulent transport, plasma stability, and plasma dynamics, see Fig. 1. These processes are determined by the parameters of the plasma such as: pressure profile for each plasma species $\left[n_{j}(r), T_{j}(r)\right]$, plasma shape, current or $q(r)$ profile, $E \times B$ velocity shear profile, and the presence and distribution of energetic particle populations. The key to obtaining the desired performance gains rests on precise control of the plasma parameters in space and time, using the various forcing functions available: Ohmic heating and current drive, fusion power, as well as 
the localized heating, current drive, and flow drive obtained from auxiliary systems beams and RF. The "advance" in Advanced Tokamak, or any other "advanced" confinement device is only partly in the cleverness of the magnetic design, it also is learning to play all these interlocking feedback loops to our advantage.

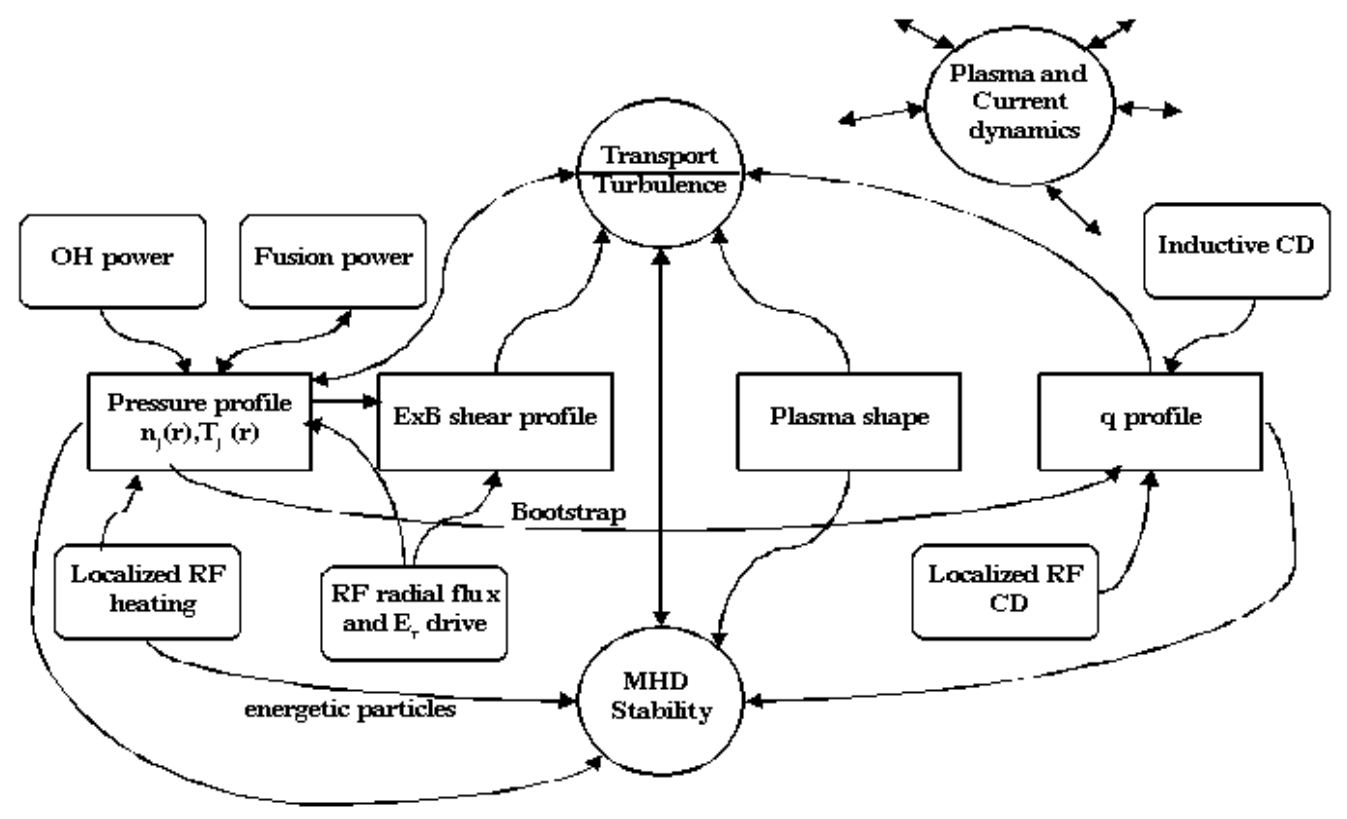

FIGURE 1. Schematic view of the various interlocking plasma processes, plasma parameters and control functions

A concrete example of the needed control is provided by reactor studies such as the ARIES-RS study (1). The configuration adopted has $\mathrm{R}_{0}=5.5 \mathrm{~m}, \mathrm{a}=1.4 \mathrm{~m}, \mathrm{~B}_{0}=8$ $\mathrm{T}$, and $\mathrm{I}_{\mathrm{P}}=11.3 \mathrm{MA}$. The plasma shape, plasma pressure and current profiles specified give high-n ballooning stability to about $\beta=5 \%$ and a bootstrap current fraction of about $f_{B S}=90 \%$. The bootstrap current differs from the required profile at the center and broadly over the outer half of the plasma. Ray tracing modeling indicates that the discrepancy can be made up by RF current drive: $5.4 \mathrm{MW}$ of ICRF for center current drive, $48 \mathrm{MW}$ of high harmonic fast wave for off axis current drive and $34 \mathrm{MW}$ of lower hybrid for edge current drive. To make such a reactor function however there are other control tasks which might well be filled by RF. A confinement improvement of $\mathrm{H}_{\text {ITER89-P }}=2.3$ is required, implying that a transport barrier of some sort must be produced. There is, however, no guarantee that the naturally occurring pressure profile will conform to that assumed for stability and consistency with the bootstrap current. Thus some methods are required to adjust the location and permeability of the transport barrier, or to directly modify the pressure profile. Furthermore rotation, or some other technique, is required to stabilize low- $n$ kink modes. 
The Spherical Torus (ST) concept faces several challenges, which present opportunities for RF (2). Because of the very small volume available for an $\mathrm{OH}$ solenoid (preferably none at all) it is desirable to heat and drive current very early in the pulse. Because ST's have low magnetic field ( $\mathrm{B}_{0} \sim 0.3 \mathrm{~T}$ in NSTX) injected beam particles have large orbits down to relatively low energies. The low field and high operating density mean that the Alfvén velocity, $\mathrm{V}_{\mathrm{A}} \sim \Omega_{\mathrm{ci}} / \omega_{\mathrm{pi}}$ is slow. Particles are therefore super-Alfvénic at modest energies. It is not yet known to what extent neutral beam heating will be compatible with ST. The low aspect ratio of ST suggests that current drive efficiency will suffer from trapped particle effects. However, ST's should be capable of very high beta, $\beta \sim 40 \%$. At such beta, a considerable portion of the plasma is in a diamagnetic well with much lower trapped particle fraction. The $\eta_{\mathrm{CD}}$ could in fact be high near the plasma center provided the high beta state can be reached. The ST constitutes a very different RF environment from the conventional tokamak and thus provides a rich field for extending RF physics. The low magnetic field means that cyclotron resonant ECH is cutoff at low density and that many closely spaced high harmonic ion cyclotron resonances will be present. On the other hand, the combination of low magnetic field and high density results in a high plasma dielectric constant so that perpendicular wavelengths are quite short $\mathrm{k}_{\perp} \sim \omega_{\mathrm{pi}} / \Omega_{\mathrm{ci}}$. This implies that absorption is strong at relatively low temperature and that very high spatial resolution is needed for modeling. In conjunction with the low magnetic field, this results in $\mathrm{k}_{\perp} \rho_{\mathrm{i}}>1$ at relatively low temperature. The propagation and absorption characteristics are, thus, quite different from our previous experience. This is also quite new territory from the standpoint of wave launching and antenna design. For example the angle between the magnetic field and the horizontal, $\theta=\tan ^{-1}\left(B_{\text {poloidal }} / B_{\text {toroidal }}\right)$ varies widely from $0^{\circ}$ at startup to $\sim 45^{\circ}$ at full current.

To achieve high performance in stellarators will require all the tricks of control needed for Advanced Tokamaks, but in 3D. Confinement in stellarators is basically anomalous. The most frequently used empirical scaling law, ISS95, is similar to Lmode in tokamaks. Reactor devices projected from this ISS95 scaling tend to be very large indeed. The production and control of transport barriers will therefore be needed, perhaps connected with the electron root of the neoclassical transport (3). Whereas we normally think of stellarators as zero current devices, the new compact stellarator concepts being considered for the U.S. program (quasi-axisymmetric stellarator and quasi-omnigeneous stellarator) do have net current. Allowing for a current profile lends added flexibility to satisfy the requirements for shear, needed for stability, magnetic configuration, and needed for improved neoclassical confinement at low aspect ratio. Although it seems to be possible to make the net current largely consistent with the bootstrap in these devices, the bootstrap profile again depends on pressure and iota so that profile control will certainly be necessary. Another consideration for near term experiments is that energetic particles are difficult to confine in non-axisymmetric devices, particularly small ones with modest magnetic 
field. Thus developing a technique for bulk ion heating such as with fundamental ICRH or perhaps IBW would be very beneficial.

Space does not permit an exhaustive discussion of all of the possible new customers for RF but we should be conscious that some of the devices commonly classified as "alternate concepts" are reaching the stage of development at which auxiliary heating and profile control could produce advances. See for example the discussion by Forrest (4) of possibilities for lower hybrid and electron Bernstein wave heating in RFP's. Our efforts and experience have been so concentrated on the conventional tokamak that we have barely scratched the surface on most other types of device. Similarly there are many applications for RF plasma techniques outside of magnetic fusion research. RF has proved to be perhaps the most saleable of plasma physics techniques. RF plasma processing of materials is a tens of billion-dollar industry, which unfortunately tries to get by with little experimental or theoretical research. RF plasmas are being studied as an approach to space propulsion (5). Finally, we should look for applications of our theory and modeling tools beyond magnetically confined plasma, to such fields as space or ionospheric plasmas and possibly to inertial fusion.

\section{ADVANCED TASKS FOR RF}

The kinds of jobs which need to be done with RF go far beyond bulk heating and current drive to much more precise and localized plasma control. For example:

- Production and control of transport barriers

- Plasma stabilization

- $\alpha$ channeling

- Production/destruction of toroidal plasma rotation

- Plasma startup/dynamics

- Thermal/configurational stability of high bootstrap current systems

The considerations for transport barriers are typical of those needed for advanced applications. Figure 2 shows schematically the processes involved in the dynamics of an internal transport barrier produced by radial shear in the $E \times B$ flow.

This figure was adapted from the work of Newman, et al. (6) on a 1D phase transition model for transport barrier formation. One can see three nonlinearly interacting feedback loops. In the principle loop the pressure gradient provides the free energy for instability drive, which produce turbulent fluctuations, which result in anomalous transport, which flattens the pressure gradient. This is the typical situation in L-mode confinement. However the diamagnetic flow associated with the pressure gradient drives a radial electric field which, if the $E \times B$ shearing rate exceeds the linear instability growth rate, can suppress the fluctuations. Also the Reynolds stress associated with the fluctuations themselves can drive flows which also produce an $E \times B$ shear. This simplified model exhibits many of the transport barrier character- 


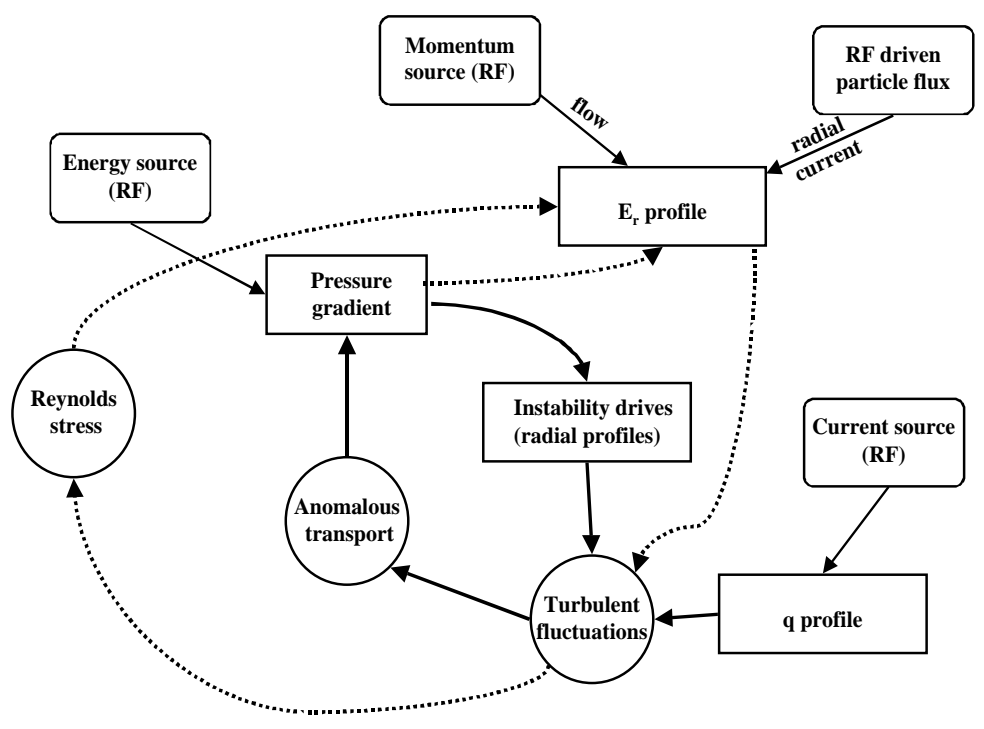

FIGURE 2. Schematic picture of the processes involved in the dynamics of an internal transport barrier produced by radial shear in the $E \times B$ flow.

istics seen in experiments: sudden transitions to low fluctuation levels, hysteresis, barrier formation near the location of minimum growth rate (e.g. near the $\mathrm{q}_{\text {min }}$ point in reverse shear) and the like. However, the mere existence of the barrier is by no means the whole point. It is necessary to trigger the barrier formation, the timing of which may be critical. One in general wishes to maximize the plasma region inside the barrier, however the resulting pressure profile must be compatible with stability and the desired bootstrap current profile. We know that in present experiments transport barrier discharges typically terminate because of uncontrolled rise in density modifying the current profile or uncontrolled rise in $\beta$ exceeding a stability limit. Results of the phase transition model suggest that important aspects of transport barriers may in fact be controllable with RF. Combinations of localized heating and fuelling may trigger transitions at power levels lower than the natural threshold. Adjusting the profile of instability growth, for example by moving the $\mathrm{q}_{\text {min }}$ point with localized current drive can control the barrier location. It may be possible to control barrier permeability by carefully operating near the transition point or by judiciously triggering back transitions to prevent excessive pressure buildup. Research in this direction constitute a major portion of the programs of DIII-D, using ECH, beams and fast waves, and Alcator C-Mod, using fast waves and lower hybrid.

For this or any of the advanced applications listed above our task is to develop the techniques and technology to make them work in experiments - routinely. Clearly we must understand the physics involved. And we must develop models which can 
calculate these processes with the quantitative accuracy and self-consistency as to definitively identify the phenomena active in experiments, and to reliably project to new applications and devices.

\section{ELEMENTS OF A COMPLETE RF THEORY}

A complete theory of RF interaction with plasmas is conveniently separated into pieces, which to a large extent can be studied independently, see Fig. 3. To be useful the pieces must be integrated together and must be integrated with models of transport and stability and with experimental data.

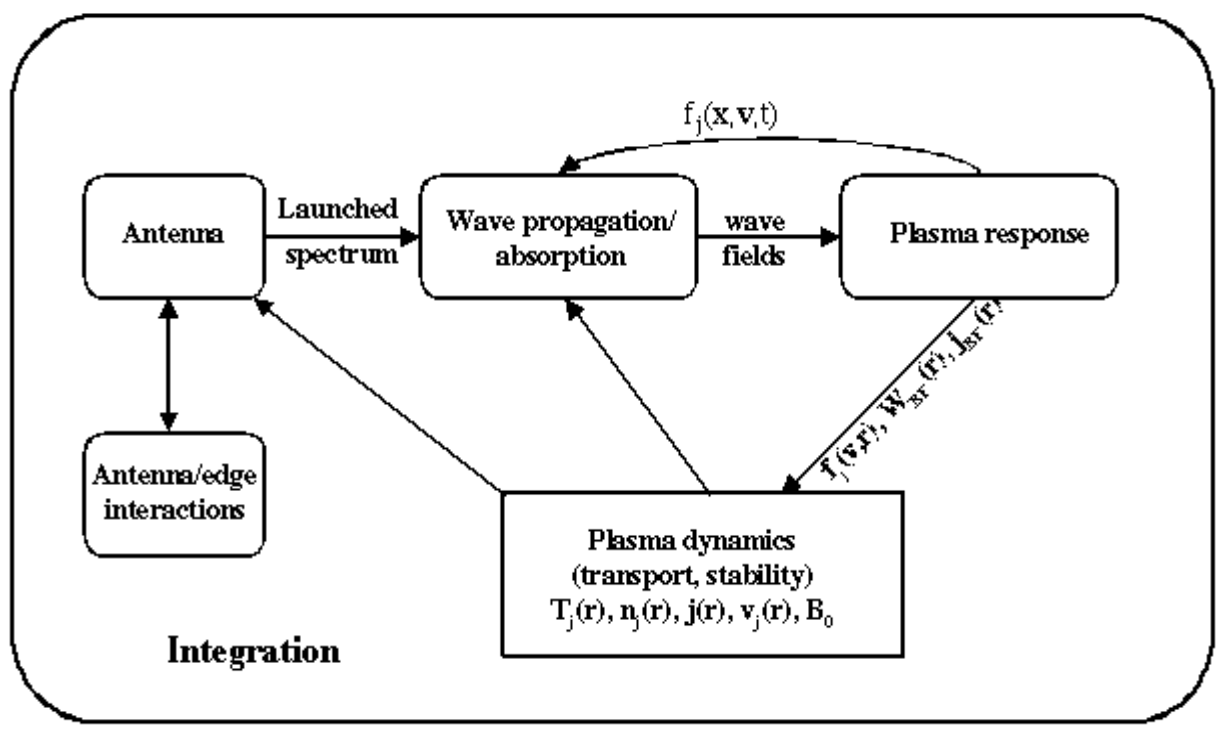

FIGURE 3. Elements of RF theory

\section{Wave Launching}

The first step in any application of RF power is to launch the correct $k$ spectrum of waves into the desired plasma mode. For electron cyclotron waves, which propagate in vacuum, the waves are launched as a narrow beam by a structure well removed from the plasma. All that remains is an essentially engineering problem of designing an antenna with the beam launch direction and polarization in the vacuum to excite the desired mode in plasma. For lower frequency waves, which propagate only above a certain plasma cutoff density, a complicated 3D launching structure must be located quite near to the plasma. Three dimensional numerical models to calculate launched wave spectra tend to come in two basic flavors: 1) Fourier models, such as RANT3D (7) or the lower hybrid grill codes (e.g. 8), where the antenna geometry is simplified to rectangular zones in each of which the wave fields are represented as a superposition of waveguide modes; and 2) finite difference or finite element E\&M codes such as 
ARGUS (9). The Fourier codes assume 1D plasma (variation in $x$ ) and calculate the power launched into each $k_{\mathrm{y}}, k_{\mathrm{z}}$ mode. Within the approximation of straight, rectilinear geometry considerable complication can be included: recessed or protruding structures, conducting septa, discrete (but usually zero thickness) Faraday shield. The finite difference codes are excellent at modeling complicated geometry: curved surfaces, straps, feeders, and finite thickness Faraday shield. However, so far it has proved difficult to couple these codes to plasma, even slab plasma, because the plasma impedance is known in Fourier space $\left(k_{\mathrm{y}}, k_{\mathrm{z}}\right)$ not locally. It has proved useful to use the two types of code together. A finite difference code, or experimental measurement, determines the current distribution on the feeders and current strap, and characterizes the action of the Faraday shield. With this as input the Fourier code can calculate the launched spectrum.

When good edge plasma profile information is available, 3D ICRH models give good agreement with values of certain integrated quantities, such as loading resistance, measured on tokamaks. Some quantities sensitive to launched spectrum, such as current driven by fast waves or lower hybrid are also well predicted by existing models. It is probably fair to say that for purposes of calculating launched spectra in tokamaks; our models are more accurate than the edge plasma density profile measurements, to which the models are sensitive. In applying models of this type to spherical tori, and particularly to stellarators, it is necessary to make severe approximations to the plasma geometry. Our experience is very limited and so far there is little experimental data with which to compare.

An even more serious issue for the technology of antennas and a more difficult issue for theory and modeling is the interaction of the antenna with the edge plasma. High amplitude RF fields near the antenna non-linearly modify the edge plasma parameters which in turn affect coupling to the desired modes. There are a number of processes involved. Ponderomotive forces tend to push the plasma away from the antenna which can decrease the coupling to desired modes and promote coupling to undesired edge modes. RF driven sheaths result in local power dissipation, which can damage the antenna and other plasma facing components, can generate impurities and decrease the available useful power. Undesirable edge modes such as slow waves or coaxial modes reduce launched power and contribute to the ponderomotive effects. Convective cells can increase the plasma flux into the antenna. Eliminating or reducing these effects is certainly one of the most critical challenges in making RF attractive and reliable at frequencies below the electron cyclotron range.

To calculate these processes is very complicated. Each one is $3 \mathrm{D}$ and is very sensitive to details of geometry. They are all nonlinearly and dynamically coupled. There has been much work done on individual elements of the problem, especially by the Lodestar and Tore Supra groups. Some trends in the rather sparse experimental data can be reproduced, such as the rate of impurity generation with power and $Z$ of the plasma facing components. However most of the work is more in the nature of 
identifying candidate effects to explain observations than the production of quantitative, predictive models. To really get a comprehensive solution probably requires a fully $3 \mathrm{D}$, electromagnetic PIC code solution in the entire antenna/edge domain, with complete geometry. This would be a very fruitful area for some basic experiments and careful validation of models, if we are serious that the U.S. program is now a science program.

\section{Propagation and absorption}

The basic RF modeling problem is to solve the linear wave equation with appropriate boundary conditions,

$$
\nabla \times \nabla \times \mathbf{E}+\frac{\omega^{2}}{c^{2}} \mathbf{E}=\mathbf{J}_{p}+\mathbf{J}_{a n t}
$$

where $\mathbf{J}_{a n t}$ is the antenna source current and $\mathbf{J}_{P}$ is the fluctuating plasma current due to the wave. The wave source can also be introduced through the boundary condition as for example an incoming wave or a non-zero tangential $\mathbf{E}$ at the boundary of the computational domain provided for example by an antenna code. Solving a $2^{\text {nd }}$ order partial differential equation (PDE) for a vector field in general three-dimensional geometry is a challenging enough computational problem, but the really hard part is obtaining the plasma current which is a 4 dimensional, non-local in space and time, integral operator on the wave field $\mathbf{E}(\mathbf{x}, t)$,

$$
\mathbf{J}_{P}(\mathbf{x}, t)=e \int d^{3} \mathbf{v} \mathbf{v} \tilde{f}(\mathbf{x}, \mathbf{v}, t) \quad \tilde{f}(\mathbf{x}, \mathbf{v}, t)=-\frac{e}{m} \int_{-\infty}^{t} d t^{\prime} \mathbf{E}\left(\mathbf{x}^{\prime}\left(\mathbf{x}, \mathbf{v}, t^{\prime}\right), t^{\prime}\right) \cdot \frac{\partial f_{0}}{\partial \mathbf{v}^{\prime}}
$$

where $\mathbf{x}^{\prime}\left(\mathbf{x}, \mathbf{v} t^{\prime}\right)$ is the orbit in the unperturbed fields of a particle which is at $(\mathbf{x}, \mathbf{v})$ at time $t$. In other words to find the perturbed distribution function $f(\mathbf{x}, \mathbf{v}, t)$ one must follow particles backward in time and weight the electromagnetic field then and there with the velocity space gradient of the time averaged distribution function $f_{0}(\mathbf{x}, \mathbf{v})$. The plasma current is then found by integrating this over velocity space.

The degree to which this problem can be solved relates to a hierarchy in the number of dimensions of the plasma and the boundary conditions which, for the problem at hand, cannot be considered ignorable. If all of the dimensions can be considered ignorable, i.e. infinite uniform plasma, then the unperturbed particle orbits are simple straight-axis circular helices and the solutions of Eq. (1) are a superposition of plane waves $\mathbf{E}(\mathbf{x}, t) \sim \exp [i(\mathbf{k} \cdot \mathbf{x}-\omega t)]$. Then all of the integrals can be carried out analytically, at least for Maxwellian $f_{0}$, and one obtains for the plasma current the textbook warm-plasma conductivity tensor involving Bessel functions of $k_{\perp} \rho$ and

plasma dispersion functions $Z\left((\omega-\ell \Omega) / k_{\|} \mathrm{v}_{t h}\right)$. Furthermore the wave equation becomes algebraic in $\mathbf{k}$ (although it is transcendental) and it can, therefore, be solved. One obtains solutions for $\mathbf{k}$ representing uncoupled eigenmodes $-\mathrm{X}$-modes, O-modes, fast waves, slow waves, IBW ... This is the source of all of our intuition about wave 
modes. A key point in this is that since the particle velocity is constant as it moves along the magnetic field and the wave phase velocity is constant everywhere, if a particle is resonant with the wave it stays resonant forever.

If the plasma has one or more non-ignorable dimensions then in general it is infeasible to solve the problem. Neither the electric field nor the unperturbed orbits have a simple form so that the time history integral in Eq. (2) cannot be done analytically. Since both $\mathrm{v}_{\|}$and the effective local value of $k_{\|}$(if such a thing can be defined) are changing as a particle moves along a field line, a particle in resonance with the wave, $\omega=\ell \Omega+k_{\|} \mathrm{v}_{\|}$, quickly goes out of resonance and becomes decorrellated. There is, however, one special case. If the plasma varies only in one dimension, $n_{e}(x), \mathbf{B}_{0}(x)$, and $\mathbf{B}_{0}$ is perpendicular to $x$ (i.e. lies in the $y-z$ plane), then again $\mathrm{v}_{\|}$ is constant and the wave field is representable as $\mathrm{E}(\mathrm{x}) \sim E(x) \exp \left[i\left(k_{y} y+k_{z} z\right)\right]$ plane waves in the $y-z$ plane. The unperturbed orbits are almost circular helices so parallel plasma response is again described by $Z$ functions. In the $x$ direction the response is still an integral operator on $E(x)$ which gives for the wave equation [Eq. (1)] a onedimensional integro-differential equation or equivalently an infinite order ordinary differential equation (ODE). We have a little experience in solving such equations using so-called integral codes such as METS95 or SEMAL (10). If one further restricts consideration to cases in which the Larmor radius is much smaller than the wavelength $k_{\perp} \rho<<1$ then $E\left(x^{\prime}\right)$ in the time history integral can be Taylor expanded $E\left(x^{\prime}\right)=E(x)+\left(x^{\prime}-x\right) E^{\prime}(x)+\frac{1}{2}\left(x^{\prime}-x\right)^{2} E^{\prime \prime}(x)+\ldots$ to some low order, typically $2^{\text {nd }}$ or $3^{\text {rd }}$. In this case the plasma response includes cyclotron harmonic interactions up to $\ell$ $=2$ or 3 and the wave equation is an ODE of order $2 \ell+2$. The solutions represent approximate eigenmodes, which are locally coupled. Our intuition concerning mode conversion comes from equations such as this.

If there are two non-ignorable coordinates, as for tokamak geometry, the third dimension can still be eliminated by Fourier transformation to obtain partial differential-integral equations in 2 variables $(r, \theta)$ or $(\psi, \theta)$, or equivalently if the wave field is expanded in poloidal modes, we get a large number of coupled ordinary differential-integral equations

$$
\mathbf{E}(\mathbf{x})=E(\psi, \theta) e^{i n \phi}=\sum_{m} E_{m}(\psi) e^{i(m \theta+n \phi)}
$$

where the equations for $E_{m}(\psi)$ are all coupled together.

For tokamak geometry there actually is a rigorous theory of the plasma response. In 1972, Kaufman (11) published a guiding-center Hamiltonian formulation of the plasma response in terms of action-angle variables, including the slow quasi-linear evolution of the equilibrium distribution function. Another approach has been developed essentially extending the slab geometry calculations described above by careful calculation of the guiding-center orbits (12) in more general geometry. It has recently been shown that the two approaches contain equivalent physics. In either case a great deal of compromising, approximating and analyzing of specific cases is 
required to obtain a formulation which is computationally feasible. These compromises are typically: restriction to low cyclotron harmonics $\ell \leq 2$, restriction to small Larmor radius, $\rho / a<<1, k_{\perp} \rho<<1$ ( $\rightarrow$ Taylor expansion of $E(\psi)$, assume orbits don't deviate much from flux surface ( $\rightarrow$ banana width $=0$ ), neglect variation of $k_{\perp}, v_{\|}$along field line ( $\rightarrow Z$ function). The physics retained after these approximations is essentially the expanded slab conductivity operator applied locally in 2D. Furthermore it is usually necessary to eliminate the Bernstein waves through order reduction techniques or to artificially damp them because of their short wavelengths. Within these compromises we do get useful results, for example in minority heating where mode conversion is not dominant or for fast wave current drive. And, of course, for short wavelength waves such as ECH or lower hybrid geometrical optics is usually adequate.

If there are no ignorable coordinates, as in stellarators, we get PDE's in 3D. Or since toroidal modes are no longer independent, expanding in both poloidal and toroidal modes gives very very many coupled ODEs for the $E_{m, n}(\psi)$.

$$
\mathbf{E}(\mathbf{x})=E(\psi, \theta, \phi)=\sum_{m, n} E_{m, n}(\psi) e^{i(m \theta+n \phi)}
$$

The guiding-center Hamiltonian formulation for plasma response does not extend directly to the stellarator case for lack of a third constant of motion equivalent to the tokamak $P_{\phi}$. Similarly it will be difficult to rigorously extend the guiding-center orbit formulation to include bounce harmonic and finite orbit width effects. Nevertheless a start has been made in applying what is effectively the local slab geometry formulation to 3D (13).

Why do we have to compromise? Finiteness of computer time, and of our own lifetime, makes us compromise on the conductivity operator. We just can't do the 4D time history/velocity space integral at every point in the computational domain for every mode. Even if the time history integral is done analytically by some asymptotic expansion, calculating the $2 \mathrm{D}$ velocity space integral $\mathrm{v}_{\|}, \mathrm{v}_{\perp}$ at every point for every mode is exceedingly expensive. Whereas evaluating the $Z$ function is fast. Finiteness of the computer size makes us compromise on resolution. Even if we can afford the time to generate the equation coefficients, the matrix size becomes too large to fit on the computer if wavelengths are very short relative to the plasma scale as in mode

converted IBW or fast waves on NSTX. We are just at the edge of the capacity to solve for fast waves in 3D even in a reduced formulation.

\section{Plasma Response - Fokker Planck Equation}

A solution for the wave fields as described above depends on the unperturbed plasma distribution function, $f_{0}(\mathbf{x}, \mathbf{v}, t)$ (a seven dimensional object), but does not give this distribution directly. Rather information about $f_{0}$ comes from the kinetic equation or Fokker Planck equation. Fortunately there is a very wide 
separation of time scales involved in the relevant phenomena so that much simplification based on judicious averaging is possible. We have: wave period or cyclotron period $1 / f_{R F} \sim 2 \pi / \Omega_{c}<10^{-7} \mathrm{sec}$, compared to particle transit or bounce times $\tau_{b} \sim 10^{4}-10^{5} \mathrm{sec}$, compared to confinement time or heating time $\tau_{E} \sim \tau_{Q L} \sim 0.1-1 \mathrm{se}$. Averaging over the very fast time scale gives the Fokker Planck equation for the gyro-averaged distribution function $f_{0}\left(\mathbf{x}, \mathbf{v}_{\|}, \mathbf{v}_{\perp}, t\right)$

$$
\frac{\partial f_{0}}{\partial t}+\left(\mathbf{v}_{\|} \hat{\mathbf{b}}+\mathbf{v}_{\mathbf{d}}\right) \cdot \nabla f_{0}=C\left(f_{0}\right)+Q\left(f_{0}\right)+S .
$$

The $2^{\text {nd }}$ and $3^{\text {rd }}$ terms on the left describe respectively particle flow along field lines and cross-field drift giving rise to radial transport. $C\left(f_{0}\right)$ describes collisional relaxation, $Q\left(f_{0}\right)$ describes the quasi-linear velocity space diffusion due to the waves, and $S$ gives particle sources and sinks. Averaging over the next most rapid time scale, transit/bounce motion, allows the dependence on the coordinate along a field line to be eliminated in terms of the velocity space coordinates $\varepsilon=$ particle energy and $\mu=$ magnetic moment, $f_{0}=f_{0}(\psi, \theta, \varepsilon, \mu, t)$. Finally in axisymmetric systems, averaging over the complete orbit allows the coupling between $\psi$ and $\theta$ to be expressed in terms of the conservation of canonical toroidal angular momentum, $P_{\phi}=m R \mathrm{v}_{\phi}+e \psi$. We are thus left with an orbit-averaged equation for $f_{0}\left(\varepsilon, \mu, P_{\phi}, t\right)$ depending on 3 constants of motion and time.

There are two general approaches to solving equations such as this. First is a direct solution as a PDE (14). The presence of finite orbit effects and radial transport make it an inherently 3D equation with non-local coefficients. In this form what typically has been done is to assume zero orbit thickness, eliminating transport or introducing ad hoc transport models, and to solve in $(\varepsilon, \mu)$ separately on each flux surface by balancing quasi-linear diffusion versus collisions $C\left(f_{0}\right)+Q\left(f_{0}\right)=0$. Useful results are obtained at modest power when large orbit effects or radial transport effects are small - ECH, lower hybrid in the absence of fast electron diffusion, or minority ICRH with weak tail.

A second approach is to solve by Monte Carlo techniques (15). One follows in time an ensemble of individual particles and periodically adds velocity increments to model the effects of RF and collisions. The guiding center orbit can be followed, making it possible to treat very complicated geometries such as stellarators, although this is a slow process. Or the orbit average of the statistical increments to the constants of motion can be calculated, allowing for a very much longer time step. Whether the orbit average approach can be extended to 3D systems having no constant of motion equivalent to $P_{\phi}$ is an open question. It is conceivable that the longitudinal adiabatic invariant $J=\oint d s v_{\|}(s)$ could serve the purpose. The Monte Carlo technique holds a great deal of promise for the future because it directly includes finite orbit and transport effects, and is much more readily parallelizable than is solution of a PDE.

An important issue is the consistency of the equilibrium distribution function used in the wave propagation calculation with the wave fields used in the Fokker Planck 
code. If the RF modified distribution function is not fed back to the wave code and the process iterated to convergence, very large discrepancies can arise. In general, this is a very complicated business. Most attempts at self-consistency have been restricted to $1 \mathrm{D}$ or to geometrical optics calculations with the quasi-linear distribution used only to calculate damping, not propagation. A beginning has been made in $2 \mathrm{D}$ with the SELFO code (16). Here a 2D finite difference, full-wave code LION has been interfaced to an orbit averaged Monte Carlo code, FIDO. Two dimensional wave fields from LION are used in FIDO to calculate $f_{0}\left(\varepsilon, \mu, P_{\phi}\right)$. This is then transformed to get a local distribution $f_{0}\left(\psi, \theta, \mathrm{v}_{\|}, \mathrm{v}_{\perp}\right)$, which is represented at each spatial grid point in terms of triangular finite elements in velocity space. The local conductivity tensor is then calculated in FIDO and fed back into LION. This combination has been successfully used to model $3^{\text {rd }}$ harmonic ICRH absorption for FWCD experiments on Tore Supra and absorption by $\alpha$ particles on JET.

It should be noted that in many cases the RF power does not cause a significant distortion of $f_{0}$ away from Maxwellian, rather it can be thought of as providing macroscopic sources to transport or stability - heat deposition, driven current, or driven plasma flow. Given the RF fields there are fairly well developed formalisms for local power deposition or driven current. However in the important area of flow drive we have only scratched the surface. Calculations of the RF poloidal force have been given (17). However to calculate the flow velocity this force is balanced against a rather ad hoc "viscous drag". What is required is a complete neoclassical formulation including toroidal geometry and proper treatment of the RF driven radial particle fluxes and ambipolar electric field.

\section{Integration}

The final box in Fig. 3 is the one surrounding all of the others - integration. Many of the limitations to what we can achieve with present models stems from our failure to put together all of the pieces. In many cases some of the pieces have been combined:

- Antenna (RANT3D) + 2D full wave (PICES) + equilibrium (VMEC)

- Current drive (FWCD, LHCD, bootstrap) + equilibrium + stability - ACCOME code

- Fokker Planck + ray tracing - several examples

- Monte Carlo + full wave - SELFO code

- Interpretive transport + RF models - e.g. TRANSP code

But it has not all been put together anywhere. A particular need is for tighter connection to experiments. It is very time consuming to get the experimental data needed as input for the RF models, even if the data exists. This is especially the case if one attempts to compare results from various devices, each having a different format and retrieval process. It was particularly informative to see the effort required to 
answer the question - how well does the RANT3D antenna code model fast wave launch in TFTR? This is, of course, only one element of the complete RF picture. To make a meaningful comparison required a team of theorists, RF experimentalists, plasma diagnosticians, and antenna technologists and required the construction of a special diagnostic to measure the edge density profile near the antenna (7). Information flow from modeling back to the experiments is not what it should be either. The most powerful of the RF models are not well coupled to the transport codes or other tools used to understand experiments. And with a few exceptions, such as predictions of fast electron bremsstrahlung measurements in LHCD experiments, the models don't calculate quantities, which can directly be compared to diagnostics.

To be concrete let us ask the question - could we make a definitive statement as to whether a given mode conversion current drive experiment agrees with theory? To do this we would need to quantitatively account for: the power and $k$ spectrum of launched fast waves; the fast wave power converted to IBW and the spectrum of IBW waves; the current driven respectively by fast waves and IBW; parasitic absorption on minorities, electrons, and beam ions; effects of equilibrium modification by heating; changes to $\mathrm{OH}$ current and heating; and the rates of all these processes. With a great deal of effort we could probably do a reasonable job on any one item. But the data needed to complete the chain is unlikely to be available either from experiment or complementary models.

\section{Future directions}

A major factor in the gaps and compromises described above is the limitation of computing power. When each one of the model elements runs up against computing limits, it doesn't seem practical to generalize the models or integrate. However we need to plan for the appearance of very much more powerful, massively parallel computers. So what does the ultimate, massively parallel RF code look like?

- High enough resolution to treat all waves of interest

- 3D

- Arbitrary $f_{0}$ self-consistently coupled to Fokker Planck model - including radial transport and finite orbit width

- General conductivity operator - high harmonics $\ell>2$, proper spatial decorrelation for the geometry

- Integrated: antenna model + propagation model + Fokker Planck + transport model + experimental data

Achieving this is not simply a matter of buying a bigger, faster computer. There is a considerable amount of rather basic, mathematical and computer science research to be done. Our present codes and experience are built on scalar or vector machines whereas the new ASCI level computers are quite different. For example, there are a number of questions relating simply to solving the matrix equations. Should we 
depend on the emergence of efficient massively parallel techniques for direct matrix inversion or should we try to develop iterative techniques, which work on our problems? The operators with which we deal are not elliptic, so iterative solutions often fail. But perhaps special preconditioning techniques could be developed to make iteration reliable

In approaching the new paradigms in super-computing, new and possibly radically different approaches to RF modeling should be considered. Perhaps time-domain solution for $\mathbf{E}(\mathbf{x}, t)$ and/or gyro-kinetic solution for $f(\mathbf{x}, \mathbf{v}, t)$ will scale the best with massive-parallelization. Is it clear that use of flux coordinates is optimum? Most particles do stay near a flux surface, but the waves don't conform to flux surfaces at all. The poloidal/toroidal mode expansions we typically use are just representations of the fields. Other representations are possible and might be advantageous, particularly in situations where the modes are coupled as poloidal modes are in 2D tokamaks and both poloidal and toroidal modes are in stellarators. Cartesian coordinates for example are simple and admit simple Fourier representations in plane waves, plus they don't have the property of concentrating resolution on the magnetic axis to an infinite degree as poloidal mode expansions do. We should also consider the issue of coordinate representation versus Fourier representation, independent of the particular choice of coordinate system. The solutions we seek generally consist of a small number of independent modes whose wavelength varies slowly in space but couple or are damped in narrow spatial regions. A coordinate representation contains no wavelength information and requires integration to recover wave/particle interactions whereas a Fourier representation contains no spatial information and relies on interference to recover spatially local features. However over the last few decades a set of mathematical concepts have been developed, wavelets and multiresolution analysis, which reveal local wavelength structure. These tools have been used in many practical applications, such as image or signal processing, but there is very little experience in using them to solve equations.

Finally what should be done about the issues of integration and communication with the experiments? A major impediment is the lack of standards for code interfaces, data exchange between codes or experimental databases and procedures for data access. This is largely an issue of convention and computer science. It is being addressed to some extent within the U.S. by the National Transport Code Colaboratory (NTCC) for which a new transport code framework is being written and consideration being given to establishing a common data format around the MDS+ system. The RF community should be aware of this project, should influence its direction, and should take advantage of any useful interfacing or standardization tools, which are developed. The largest new opportunity for super-computing in plasma physics in the US will come through the Strategic Simulation Initiative (SSI) if the fusion proposal is successful. Unfortunately as presently conceived (April, 1999) the fusion SSI proposal is narrowly focused on gyro-kinetic simulation of turbulence and 
possibly non-linear MHD to the total exclusion of any support for RF theory and modeling. It was apparently the judgement of the fusion SSI steering committee that we are not ready.

\section{ACKNOWLEDGEMENTS}

The author would like to thank a large number of colleagues who provided material or offered suggestions for this talk. This work was supported by U.S. Department of Energy Contract No. DE-AC05-96OR22464 with Lockheed Martin Energy Research Corporation.

\section{REFERENCES}

1. Jardin, S. C., et al., Fusion Eningeering \& Design, 38, 27 (1997).

2. R. Majeski, et al., this conference.

3. Erckman, V., et al., this conference.

4. Forest, C. B., this conference.

5. Baity, F. W., et al., this conference.

6. Newman, D. E., et al., Physics of Plasma 5, 938 (1998).

7. Carter, M. D., et al., this conference.

8. Brambilla, M., Nucl. Fusion, 16, 47 (1975). See also Bers, A., and Teilhaber, K. S., Nucl. Fusion 23, 41 (1983).

9. Ho, Y. L., et al., 10 th Topical Conf. on RF Power in Plasmas, AIP Conf. Proc. 289 (Boston, MA, 1993) 359. See also Vecci, et al., this conference.

10. Smithe, D., report MRC/WDC-R-363 (1995). See also Sauter, O. and Vaclavik, J., Computer Physics Communicating 84, 226 (1994).

11. Kaufman, A. N., Phys. Fluids, 15, 1063 (1972).

12. Lamalle, P. U., Plasma Physics Control. Fusion, 40, 465 (1998). See also Kock, R., Lamalle, P. U. and Van Eister, D., Plasma Physics Control. Fusion, 40, A191 (1998).

13. Vdorin, V., Watari, T., and Fukuyama, A., Proc. of ICPP96, Nagoya (1997), 1070.

14. Kerbel, G. D., and McCoy, M. G., Phys. Fluids, 28, 3629 (1985).

15. Carlsson, J., Erickson, L.-G., and Hellsten, T., Proceedings of the Joint Varenna-Lausanne Workshop "Theory of Fusion Plasmas", Bologna: Editrice Compositori (1994), 351-356.

16. Hedin, J., Hellsten, T., and Carlsson, J., Proceedings of the Joint Varenna-Lausanne Workshop "Theory of Fusion Plasmas", Bologna: Editrice Compositori (1998), 467-472.

17. Berry, L. A., Jaeger, E. F., and Batchelor, D. B., Phys. Rev. Lett. 82, (1999) 1871. See also, Myra, J. R. and D'Ippolito, D. A., this conference. 